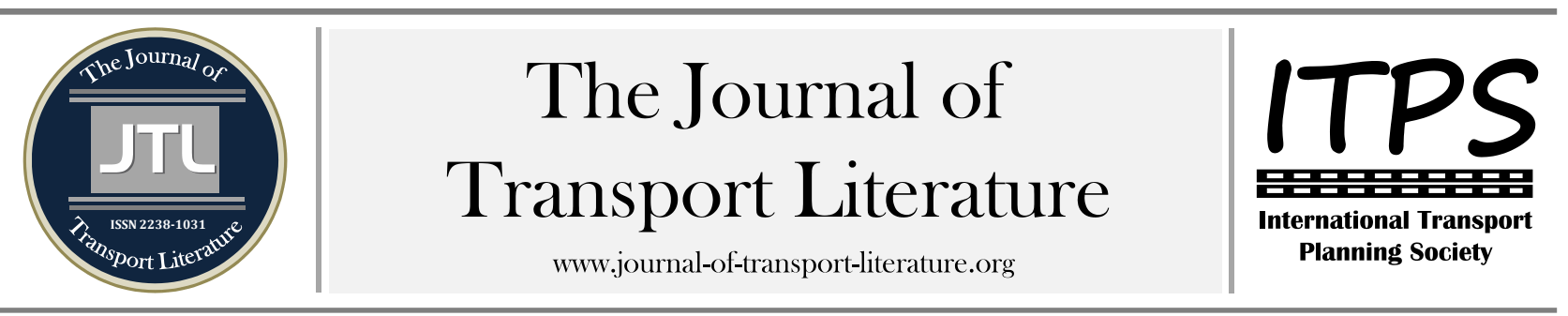

\title{
Modelo de estimação de custos do transporte urbano de cargas com base na vulnerabilidade da rede viária
}

\author{
George Vasconcelos Goes ${ }^{+}$; Bruno Vieira Bertoncini
}

Universidade Federal do Ceará, Fortaleza, Brasil

\section{Article Info}

Palavras-chave: rede de transporte vulnerabilidade risco

Submitted 17 Apr 2015 form 16 Jul 2015; accepted 31 Aug 2015

Licensed under

Creative Commons

CC-BY $3.0 \mathrm{BR}$

\section{Resumo}

Vulnerabilidade é um atributo de desempenho que busca inferir o impacto que a obstrução total ou parcial de uma via reflete no desempenho da rede de transportes. Tal conceito permite analisar tanto a resiliência da rede como os custos incorridos em cenários distintos para diversos atores envolvidos. Este artigo objetivou propor uma metodologia de análise e mensuração dos custos da vulnerabilidade associada ao transporte de cargas. Para isso, desenvolveu-se um modelo de rede viária com 15 arcos e 10 nós, baseado no equilíbrio do usuário, com a finalidade de realizar simulações de falhas nos arcos, além de mensurar os riscos de haver obstruções. 0 modelo contendo os custos e risco por cenário apresentou desempenho satisfatório para a representação do fenômeno e para a tomada de decisão.

+ Corresponding author. Universidade Federal do Ceará, Departamento de Engenharia de Transportes. 60455-760 - Fortaleza, CE - Brasil.

E-mail address: george.vasconcelos@outlook.com.

\section{Introdução}

Os problemas de mobilidade urbana estão relacionados, dentre outros fatores, à distribuição espacial das atividades, ao crescimento expressivo do uso do automóvel, associado a um sistema deficiente de transporte público, e à ocorrência de impactos negativos provocados pelas atividades de movimentação de cargas nas áreas adensadas (Sanches Júnior, 2008); cujos impactos ocasionados pelo trânsito possuem maiores proporções (sociais e ambientais). A concentração populacional nas cidades confere aos centros urbanos papel de núcleos de consumo, que devem ser abastecidos continuamente a partir de fluxos de natureza e origem muito diversas. Apesar de tamanha relevância, a discussão sobre distribuição de carga urbana tem ocupado um lugar secundário dentre as prioridades de planejamento conduzido pelas autoridades (Muñuzuri et al., 2005; Macário et al., 2006; Quack e De Koster, 2008).

Eventos como a enchente no Estado do Acre (2014) e o terremoto em Kobe (2005), cujas regiões sofreram interrupções no abastecimento de produtos essenciais, são de baixa probabilidade de ocorrência, contudo o custo generalizado decorrente para os envolvidos, direta e indiretamente, pode ser suficientemente elevado ao ponto de legitimar pesquisas acerca de atributos como vulnerabilidade de redes de transporte. Para D’Este e Taylor (2001) o propósito de analisar a vulnerabilidade de redes de transporte se baseia na antecipação aos pontos de fraqueza onde essas redes são altamente vulneráveis, ou seja, falhas nos nós e arcos resultarão em efeitos adversos substanciais nos seus desempenhos, em particular nas estratégias de escolha de rota e distribuição. Diante disso, a literatura especializada aponta autores como Taylor et al. (2006), Chen et al. (2012), Knoop et al. (2012), Scott et al. (2006), Snelder et al. (2012) entre outros, cujas pesquisas buscam investigar os atributos de desempenho da rede de transporte como forma de controlar os custos específicos ou generalizados oriundos da sua utilização. 0 conceito de vulnerabilidade é expresso como a suscetibilidade a incidentes que podem reduzir consideravelmente a capacidade da rede (Berdica, 2002). Destarte, mensurar a vulnerabilidade seria equivalente a medir o impacto que a obstrução total ou parcial, ocasionada por fatores naturais ou não de uma via, reflete no desempenho da rede de transportes. No caso das áreas urbanas adensadas, suas vias sofrem interferência de obras e manutenções que bloqueiam, total ou parcialmente, arcos da rede e impõem aos transportadores custos adicionais devido à presença de tais intervenções.

O objetivo central deste trabalho é propor uma metodologia para análise e mensuração dos custos por meio do conceito de vulnerabilidade associada ao transporte de cargas, a partir simulações de cenários. Seus objetivos específicos, com a correspondente organização do texto, são: (i) identificar no estado da arte como são abordados os modelos de confiabilidade e vulnerabilidade de links das redes de transporte; (ii) desenvolver um modelo que simule a vulnerabilidade dos links de uma rede dado um cenário de degradação. Por fim, as conclusões da análise, com recomendação de questões pertinentes ainda à serem investigadas.

\section{Custos e vulnerabilidade}

0 custo refere-se à parte do ônus que uma entidade precisa arcar para atingir seus objetivos, tais como a utilização de um produto ou serviço visando à obtenção de outros bens ou serviços. Tais custos podem ser classificados em dois aspectos: 
monetários e não monetários. Os custos podem, também, ser interpretados a partir do conceito de vulnerabilidade em que são identificados e analisados sob a forma de indicadores. Para Oliveira et al (2013), a função dos indicadores de vulnerabilidade é, medir, de forma objetiva, o quão vulnerável é uma rede viária ou, então, medir a importância de seus componentes (arcos e nós) para a sua vulnerabilidade. Em relação às variáveis quantificadas, as principais consideradas são o tempo e o custo de viagem, aumentados quando da interrupção total ou parcial de um arco.

A interrupção total ou parcial de um link decorrente de uma falha na via expõe a capacidade do sistema de transporte de operar continuamente sob condição de pressão ou risco. À depender da vulnerabilidade da rede, a região afetada pode sofrer com a interrupção no abastecimento de uma cadeia de suprimentos, onde os custos associados à reprogramação e redirecionamento dos veículos de carga podem ser proibitivos para alguns fornecedores e operadores logísticos (Scott et al., 2006). Segundo Tampère et al. (2007), a maneira de descobrir quais arcos de uma rede de transporte são vulneráveis é através da simulação de um incidente em cada link dessa rede. A próxima etapa baseia-se em computar o impacto desse incidente, por exemplo, em termos de tempo de viagem perdido. Nicholson (2003) complementa na simulação de vulnerabilidade o atributo de risco de falha do arco, ou seja, a probabilidade de ocorrência dessa interrupção.

\section{Modelos de vulnerabilidade aplicados à rede de transportes}

Taylor et al. (2006) formularam um modelo baseado no conceito de custo generalizado. Entende-se como custo generalizado impedâncias relacionadas a viagens entre pontos como: distância, tempo, dinheiro etc. Busca-se determinar a medida de vulnerabilidade da rede mediante mudança desse custo entre dois pontos decorrente de uma falha em um ou mais arcos. Em termos numéricos, considerou-se uma rede $\mathrm{G}(\mathrm{N}, \mathrm{E})$, onde $\mathrm{N}$ é um conjunto de n nós e $\mathrm{E}$ um conjunto de $\mathrm{m}$ diferentes arcos. Associado a cada arco existe um atributo de custo não negativo. Ainda segundo os autores, s[ij, $\mathrm{G}(\mathrm{N}, \mathrm{E})] \mathrm{se}$ refere ao caminho de menor custo de i a j. Isto posto:

$$
\text { vijrs }=s[i j, G(N, E)]-s[i j, G(N, E-\operatorname{ers})]
$$

\section{Em que}

$\mathrm{N}$ : conjunto de $\mathrm{n}$ nós; E: conjunto de m links; e: link comprometido.

A função refere-se à diferença entre o caminho de menor custo intacto e o caminho de menor custo sem o link que conecta o nó $\mathrm{r}$ ao nó s (ers). A relação é negativa devido a representar um incremento no custo da viagem na rede degradada. Jenelius et al. (2006) desenvolveram um modelo utilizando conceito semelhante, onde o custo da interrupção do link é determinado mediante confrontação do custo entre os nós i e j quando o elemento e falha, $c i j(e)$, e o custo inicial, sem falha, $c i j^{(0)}$.

Jenelius et al. (2006) consideram custo generalizado em seu modelo de vulnerabilidade como os tempos de viagem entre os nós demandados. Os autores assumem como simplificação que os tempos de viagem são independentes do carregamento dos arcos da rede de transportes. Taylor et al. (2006) adotam variável semelhante, onde para os autores, os tempos de viagem podem ser utilizados como uma medida da mudança do custo generalizado. Sullivan et al. (2010) adotam o tempo de viagem total como método para identificação dos links mais críticos da rede de transportes, onde se simulam interrupções no fluxo dos arcos para averiguar o impacto em termos de tempo. A literatura sugere que alterações no tempo de viagem de cada arco representam variações proporcionais do valor do custo generalizado da rede de transportes. Para Yang et al. (2000) medidas baseadas em variabilidade de tempos de viagem são úteis para avaliar o desempenho da rede de transportes em termos de qualidade do serviço prestado aos usuários no dia a dia.

\section{Método}

A abordagem metodológica apresentada nesta pesquisa possui alicerce no conteúdo descrito em Jenelius et al. (2006). Nesta circunstância, assume-se que no modelo de vulnerabilidade da rede a demanda é inelástica, ou seja, considera-se que a falha no arco leva tempo suficiente para um novo equilíbrio de usuários se formarem, contudo é curto o suficiente para não afetar a demanda de viagens de forma significativa.

Consideram-se também as etapas de alocação das viagens descritas por Oliveira et al. (2013) e adicionam-se as atribuições de custos monetários da vulnerabilidade, conforme ilustrado na Figura 1. Desta forma, conjectura-se o cenário de custos da rede de transporte pré-incidente e confronta-o com o cenário pós-incidente, no qual o saldo obtido em valores não monetários (no caso tempo) é o atributo de vulnerabilidade da rede. Subsequente à determinação do atributo não monetário de vulnerabilidade obtém-se o impacto desta em valor monetário através da mensuração do custo do tempo para o transportador.

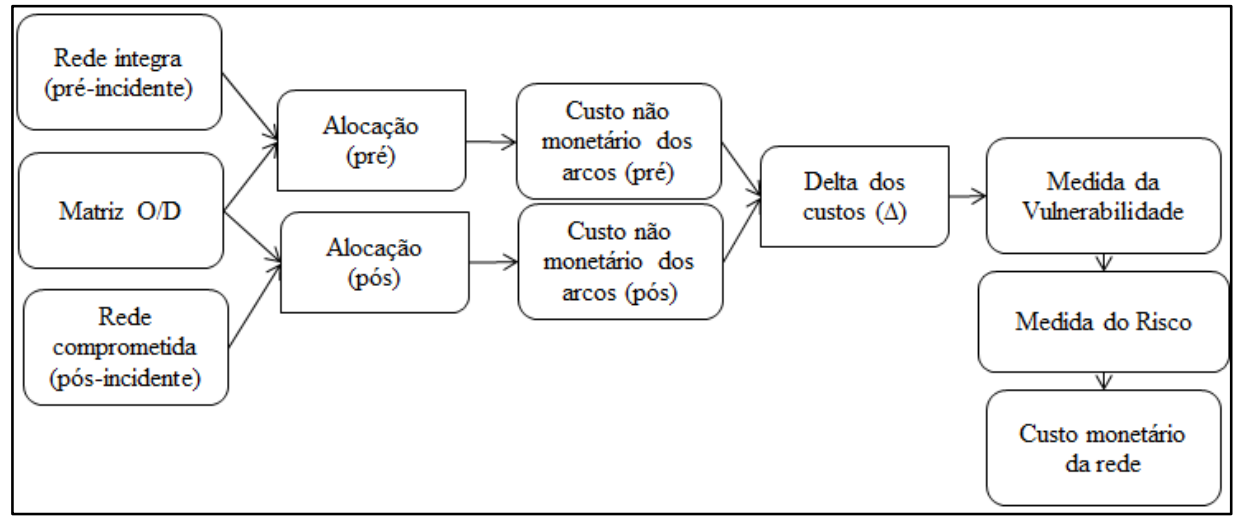

Figura 1: Etapas metodológicas para mensuração dos custos da vulnerabilidade

Na modelagem realizada, assume-se que um evento ocasiona falha em um ou mais arcos da rede de transportes. Tal evento ocasiona interrupção total do arco, o que obriga a todos os viajantes deste arco a tomar outras rotas menos 
vantajosas. Assume-se, do mesmo modo, que os usuários da rede de transportes se comportam consoante ao método de equilíbrio determinístico do usuário. Diante disso, o custo de viagem entre cada par origem-destino (OD) pós-alocação é definido de forma única. Dessa maneira, o custo inicial, sem alocação, não varia entre os arcos. A formulação é definida na Equação 3, função desenvolvida pelo Bureau of Public Roads (BPR), tratada na NCHRP 365 (TRB, 1988).

$$
c i=c 0, i\left(1+a 1_{c a p i}^{f i}\right)^{y}
$$

Em que

ci: custo do arco i;

co,i: custo inicial do arco i;

fi: fluxo no arco i;

Capi: capacidade do arco i;

y: constante calibrada;

a: constante calibrada.

No modelo de equilíbrio determinístico considerado, os fluxos dos arcos $\mathrm{f}$ e os seus correspondentes custos c são obtidos ao aplicar simultaneamente o Modelo de Oferta e Modelo de Demanda expostos por Cascetta, (2009). A Função Objetivo busca minimizar o tempo total (C) da rede de transportes de forma em que esteja em equilíbrio, conforme Equação não linear 4.

$$
\begin{aligned}
& F=\min =\sum_{i} \int_{0}^{f i} c i(y i) d y \\
& f \in S f
\end{aligned}
$$

Onde:

ci: custo do arco i;

fi: fluxo no arco i;

y: constante calibrada.

O custo oriundo da interrupção de um arco, então, segue a formulação adotada por Jenelius et al. (2006). Assim, confrontam-se os custos dos arcos com falha do elemento e $\left(c 1^{(e)}\right)$ e o custo inicial, sem falha $\left(c i j{ }^{(0)}\right)$, obtendo-se o delta de desempenho. Por fim, o risco de falhas na rede será estimado baseado no modelo de previsão de acidentes (MPA) por trecho desenvolvido por Cardoso e Goldner (2007), assumindo-se que as interrupções são ocasionadas devido a ocorrência de acidentes. As variáveis de entrada no modelo foram: volume e velocidade por arcos, onde os valores são codificados em uma base comum.

\section{Experimento}

Adotou-se nesta pesquisa um experimento hipotético de forma a obter maior controle e flexibilidade sobre as variáveis de entrada do modelo. A rede viária urbana, ilustrada na Figura 2, é composta por 10 nós, conectados entre si por 15 arcos orientados. Os pares origem-destino são representados como: 1-3, 1-4, 2-3 e 2-4.

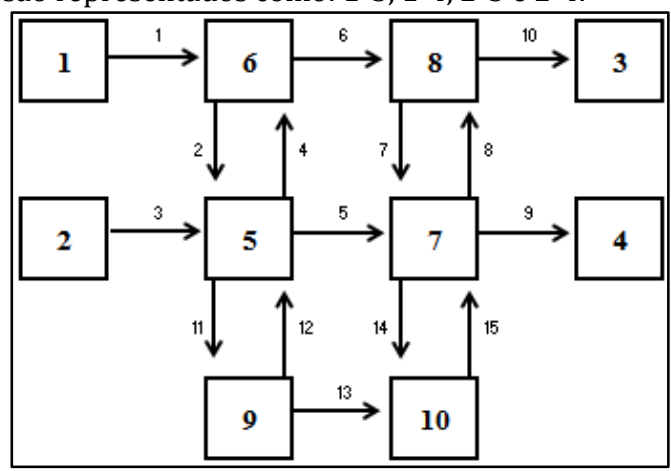

Figura 2: Representação da rede viária

A matriz origem-destino evidenciada na Tabela 1, representa o desejo de deslocamento entre os pares na rede de transportes utilizada. Desta forma, alocou-se o volume no modelo de forma a obter o status de equilíbrio das viagens e os custos dos arcos no cenário de pré-incidente.

Tabela 1: Matriz origem-destino (OD)

\begin{tabular}{llll}
\hline Origem/destino (nós) & 3 & 4 & $\sum$ \\
\hline 1 & 2.000 & 2.500 & 4.500 \\
2 & 2.500 & 1.500 & 4.000 \\
$\Sigma$ & 4.500 & 4.000 & 8.500 \\
\hline
\end{tabular}

Para efeito da vulnerabilidade da rede de transportes, considerar-se-á como falha uma redução drástica na capacidade do arco (Berdica, 2002). Isto posto, os arcos considerados com falhas terão sua capacidade restrita a cem veículos no instante da alocação. Para efeito da simulação dos gastos monetários, adotou-se a cotação média do diesel (R\$2,20) utilizado por veículos urbanos de carga (VUC), bem como o consumo médio $(6 \mathrm{~km} / \mathrm{L})$ desse veículo. A variação das 
características dos arcos ocorre somente na capacidade. Adotou-se o padrão de 1.250 veículos por faixa, variando em quantidades de faixas para cada arco.

Dessa forma, busca-se simular uma rede viária cujos arcos pertencentes a rotas mais curtas tenham somente uma faixa para escoamento do fluxo e os arcos que representem alternativas de maior distância possuam três faixas para escoar o volume de veículos de forma que a rede fique equilibrada. Nos demais arcos adotaram-se duas faixas como padrão de capacidade. 0 tempo total da rede é calculado mediante o somatório dos tempos individuais de cada arco.

Uma vez arquitetada a rede de transportes e as restrições do modelo, realizaram-se testes experimentais considerando seis cenários de restrições no fluxo de arcos. Os cenários simulados buscam representar as seguintes: (i) rede sem falhas; (ii) falha em um arco de uma faixa somente (dois cenários simulados); (iii) falhas em dois arcos, de uma faixa e de três faixas, (dois cenários simulados); e, finalmente, (iv) falha em um arco de três faixas (um cenário simulado). Busca-se no experimento representar um escopo abrangente de situações de eventos com baixa probabilidade de ocorrência e de impactos variados no tempo, custo e volume observado da rede, conforme:

a) Cenário 1: representa a situação em que a rede opera sem falhas nos arcos;

b) Cenário 2: neste cenário é simulada uma falha no Arco 5, assim reduz-se sua capacidade de 1.250 para 100 veículos;

c) Cenário 3: objetiva-se analisar os efeitos no tempo de uma falha no Arco 6;

d) Cenário 4: objetiva simular redução na capacidade de 3.750 para 100 veículos no Arco 13;

e) Cenário 5: neste cenário ocorrem falhas em mais de um arco (6 e 15);

f) Cenário 6: semelhante ao cenário anterior, contudo as falhas ocorrem nos arcos 5 e 13.

\section{Resultados}

Observa-se na Tabela 2 as medições do custo total em tempo, custo total em valores monetários (em relação ao consumo de combustível) e variação do tempo em relação ao status quo, ou seja, a rede viária não comprometida.

Tabela 2: Variação do custo total observado

\begin{tabular}{lllllll}
\hline Cenários & Volume & Ct (tempo) & Delta $(\Delta \mathrm{t})$ & $\%$ & Consumo & Risco \\
\hline Cenário 1: rede sem falhas & 37.509 & 599,05 & - & - & $\mathrm{R} \$ 68.766,13$ & 326,30 \\
Cenário 2: falha no arco 5 & 41.350 & 709,11 & 110,06 & $18 \%$ & $\mathrm{R} \$ 75.808,79$ & 325,37 \\
Cenário 3: falha no arco 6 & 44.859 & 780,22 & 181,17 & $30 \%$ & $\mathrm{R} \$ 82.240,96$ & 345,99 \\
Cenário 4: falha no arco 13 & 31.203 & $1.613,59$ & $1.014,54$ & $169 \%$ & $\mathrm{R} \$ 57.205,27$ & 323,58 \\
Cenário 5: falhas nos arcos 6 e 15 & 34.598 & $12.639,25$ & $12.040,20$ & $2.010 \%$ & $\mathrm{R} \$ 63.430,54$ & 290,52 \\
Cenário 6: falhas nos arcos 5 e 13 & 32.327 & $12.600,58$ & $12.001,52$ & $2.003 \%$ & $\mathrm{R} \$ 59.266,18$ & 281,36 \\
\hline
\end{tabular}

0 resultado de menor impacto no tempo total do carregamento da rede viária (pós-incidente) fora obtido no Cenário 2. Ao considerar falha no Arco 5, de menor capacidade (1.250), o tempo total no sistema aumenta em 110,06 minutos. Comparando com o Cenário 1, o custo monetário dessa falha para o transportador sobe em $10 \%$, haja vista que será necessário escolher rotas mais longas que minimizem o tempo do trajeto. 0 volume observado (somatório da quantidade de veículos que passam por cada arco) aumenta. No contexto geral, o Arco 5 indica baixa vulnerabilidade para a rede viária, apresentando menor aumento no tempo de trajeto. 0 cenário 3, busca comparar a ocorrência de falha em outro arco que possua uma única faixa (arco 6) com o Cenário 2. Novamente o volume total observado e o tempo aumentam (em 30\%), assim como o custo (19\%). Nesse cenário, os aumentos foram ligeiramente superiores ao do cenário anterior em razão de Arco 6 possuir um volume alocado superior ao Arco 5.

Na ocorrência de falha em um arco de três faixas e distante (Arco 13), conforme cenário 4, o volume observado torna-se o menor do experimento e o tempo total de trajeto aumenta em 169\%. Entretanto o custo em combustível diminui (-17\%). Esse resultado evidencia que falhas em arcos distantes acarretam em redução no volume observado e no gasto com combustível. A explicação para esse fato é que as opções de escolha de caminhos que mantêm a rede em equilíbrio são limitadas, fazendo com que mais veículos utilizem rotas com menos arcos. Quando a falha ocorre em dois arcos da rede de transportes, tanto o volume observado como o custo reduzem (cenários 5 e 6), entretanto o tempo total do trajeto aumenta exponencialmente. Observa-se, com isso, que o volume total observado e o custo em combustível não aumentam ou diminuem de forma diretamente proporcional à quantidade de arcos inutilizados da rede viária, mas sim, em relação à distância a ser percorrida pelas novas rotas resultantes disso.

Em relação ao comportamento do risco de falha (por acidentes) de cada cenário, observa-se que os maiores índices ocorrem quando as falhas são ocasionadas nos arcos de menor capacidade. Evidencia-se com isso que devido o volume ser alterado para os arcos de maior capacidade $(11,13$ e 15) os fatores de risco (velocidade e volume) são potencializados, aumentando assim o risco de acidentes. 0 inverso ocorre quando os arcos de maior capacidade são interrompidos. Apesar de gerar maior volume ou congestionamento nos arcos menores, o que aumenta o risco de colisões, a velocidade é bastante afetada. Nesse contexto há compensação no risco de acidentes, tornando o índice inferior ao status quo do Cenário 1.

A tomada de decisão pelo transportador, então, considera o trade-off existente entre o trajeto cuja falha de um arco acarreta em um menor tempo em relação aos demais e o trajeto que representa o menor custo caso um arco seja vulnerável. A Figura 3 ilustra o comportamento dos custos em cada caminho de cada cenário simulado. Os cenários com maior delta de tempo apresentam custos de locomoção menores por representar uma rede com opções de menores distancias percorrida. 


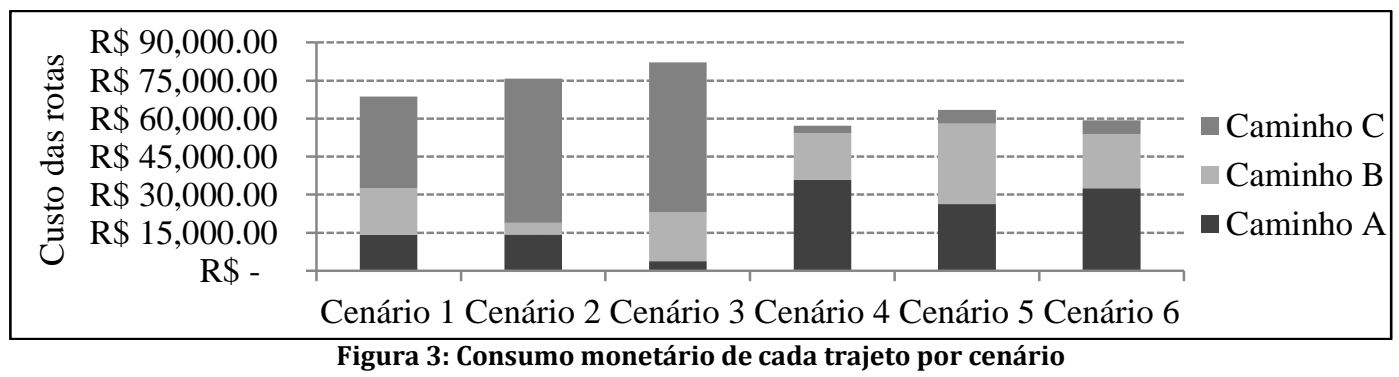

\section{Conclusões}

O experimento levado a efeito demonstra a necessidade de desenvolver estratégias de contingência pelos transportadores para eventuais interrupções nos fluxos de arcos de maior volume e com poucas ou nenhuma rota alternativa vizinha. Em especial, anseia-se quanto à criação de rotas alternativas para escoamento desse fluxo de forma que o custo total não seja alterado de forma abrupta, ou conforme Nicholson et al. (2003), melhorar a confiabilidade dos componentes (fortalecer os arcos) e monitorar os elementos críticos da rede. Para o usuário transportador, o processo de escolha da rota pode ser influenciado (à medida que aumenta o valor do consumo do combustível ou do tempo de trajeto) pela vulnerabilidade do caminho adotado. As evidências demonstram que o risco associado à ruptura de cada arco pode ser um critério de decisão de rotas ou de medidas do agente governamental quando a links prioritários em ações de prevenção.

Quanto aos objetivos, a metodologia utilizada fora eficaz em seu propósito. Desta forma, fora desenvolvido e testado um método para estimação do custo da vulnerabilidade através de simulações de cenários (objetivo geral). Abordou-se também na literatura como os modelos são tratados no estado da arte em pesquisa de vulnerabilidade e, finalmente, desenvolveu-se o conceito em um modelo matemático. Como continuidade da pesquisa, sugere-se: (i) inserir algoritmos que estimem a confiabilidade no modelo para efeito de decisão do usuário; (ii) aprofundar no conceito de escolha de rotas por custo monetário e (iii) inserir um método de escolha multicritério baseado em confiabilidade, risco e vulnerabilidade das rotas.

\section{Referências}

Berdica, K. (2002). An introduction to road vulnerability: what has been done, is done and should be done. Transport Policy, v. 9, n. 2, p. $117-127$.

Cardoso, G; Goldner, L. (2007). Desenvolvimento e aplicação de modelos para previsão de acidentes de trânsito. TRANSPORTES, v. XV, n. 2, p. 43-51.

Cascetta, E. (2009) Transportation System Analysis: models and applications. 2nd edition. Springer.

Chen, L., Miller-Hooks, E. (2012). Resilience: an indicator of recovery capability in intermodal freight transport, Transportation Science, v. 46, n. 1, p. 109-123.

D’Este, G., Taylor, M. (2001). Modelling network vulnerability at the level of the National strategic transport network. Journal of the Eastern Asia Society for Transportation Studies. Vol. 4. No. 2. October.

Jenelius, E., Petersen T., Mattson, L. (2006). Importance and exposure in road network vulnerability analysis, Transportation Research Part A, v. 40, n. 7, p 537-560.

Knoop, V. L., Snelder, M., Van Zuylen, H. J. Hoogendoom, S. P. (2012). Link-level vulnerability indicators for real world networks, Transportation Research Part A, v. 46, n. 5, p. 843-854.

Macário, R.; Filipe, L. N.; Reis, V. (2006). Mobilidade urbana sustentável e a distribuição de mercadorias. In: Congresso Luso Brasileiro para o Planejamento, Urbano, Regional, Integrado e Sustentável - Pluris, 2.

Muñuzuri, J., Larrañeta, J., Onieva, L., Cortés, P. (2005). Solutions applicable by local administrations for urban logistics improvement. Cities, Vol. 22, $\mathrm{n}^{\circ} 1$, p. 15-28.

Nicholson, A. (2003). Transport network reliability measurement and analysis, Revista Transportes, v. 11, p. 49-62.

Oliveira, E., Portugal, L., Porto, W. (2013). Determinando links críticos em uma rede viária: indicadores de Vulnerabilidade. Anpet.

Quak, H.; De Koster. (2008). Delivering goods in urban areas: how to deal with urban policy restrictions and the environment. Transportation Science, p. 1-17.

Sanches J, P. F. (2008). Logística de carga urbana: uma análise da realidade brasileira. Campinas, SP: UNICAMP.

Scott, D. M., Novak, D.C., Aultman-Hall, L., Guo, F. (2006). Network Robustness Index: A new method for identifying critical links and evaluating the performance of transportation networks, Journal of Transport Geography, v. 14, n. 3, p. 215-227.

Snelder, M., VanZuylen, H. J., Immers, L. H. (2012). A framework for robustness analysis of road network for short term variations in supply, Transportation Research Part A, v. 46, n. 5, p. 828-842.

Sullivan, J.L., Novak, D.C., Aultman-Hall, L., Scott, D.M. (2010). Identifying critical road segments and measuring system-wide robustness in transportation networks with isolating links: a link-based capacity-reduction approach. Transportation Research Part A 44, $323-$ 336.

Tampère, C. M. J., Stada, J., Immers, B. (2007). Methodology for identifying vulnerable sections in a national road network, disponível em: www.mech.kuleuven.be/cib/verkeer/dwn/pub/P2007C.pdf.

Taylor, M. A. P., Sekhar, S. V. C. e D’Este, G. M. (2006). Application of accessibility based methods for vulnerability analysis of strategic road networks. Network and Spatial Economics, v. 6, n. 3-4, p. 267-291.

Yang. H. Lo, H.K. and Tang, W.H. (2000). Travel time versus capacity reliability of a road network. In M.G.H. Bell and C. Cassir (eds.), Reliability of Transport Networks. Research Studies Press, Baldock, Herts. 119-138.

\section{Abstract}

Vulnerability is a performance attribute that seeks to infer the impact of partial or total blockage of a pathway reflects the performance of transport network. This concept allows to analyze both of the network resilience and the costs in different scenarios for the various actors involved. This article aims to propose a methodology for analysis and measurement of costs associated with the vulnerability of freight transportation. Thus, we developed a model of road network with 10 nodes and 15 links, based on user equilibrium, in order to perform simulations of failed arcs, in addition to measuring the risks of having obstructions. The model containing costs and risk by scenario sufficient to allow the representation of the phenomenon and the decision-making performance.

Key words: transport network, vulnerability, risk. 